Short Communication

Human and Medical Genetics

\title{
Leigh syndrome in a patient with a novel C12orf65 pathogenic variant: case report and literature review
}

Eduardo Perrone $e^{1,4}$ [ , Thiago R. Cavole ${ }^{1}$, Manuella G. Oliveira ${ }^{1}$, Luiza do A. Virmond ${ }^{1}$, Marina de França B. Silva ${ }^{1}$, Maria de Fatima F. Soares ${ }^{2}$, Simone Brasil de O. Iglesias ${ }^{3}$, Ariane Falconi ${ }^{4}$, Juliana S. Silva ${ }^{4}$, Viviane Nakano ${ }^{4}$, Maria Fernanda Milanezi ${ }^{4}$, Carmen Silvia C. Mendes ${ }^{3}$, Marco Antonio Curiati ${ }^{3}$ and Cecília Micheletti ${ }^{3}$

${ }^{1}$ Universidade Federal de São Paulo, Departamento de Genética Médica, São Paulo, SP, Brazil.

${ }^{2}$ Universidade Federal de São Paulo, Departamento de Radiologia, São Paulo, SP, Brazil.

${ }^{3}$ Universidade Federal de São Paulo, Departamento de Pediatria, São Paulo, SP, Brazil.

${ }^{4}$ GeneOne, Dasa, São Paulo, SP, Brazil.

\begin{abstract}
Leigh syndrome is an early onset progressive disorder caused by defects in mitochondrial oxidative phosphorylation. Pathogenic variants in nuclear and mitochondrial genes are associated with the syndrome. Homozygous pathogenic variants in the C12orf65 gene impair the mitochondrial oxidative phosphorylation system. We describe a new case of Leigh syndrome caused by a novel pathogenic variant of the C12orf65 gene resulting in the lack of the Gly-Gly-Gln (GGQ) domain in the predicted protein, and review clinical and molecular data from previously reported patients. Our study supports that the phenotype caused by C12orf65 gene variants is heterogeneous and varies from spastic paraparesis to Leigh syndrome. Loss-of-function variants are more likely to cause the disease, and variants affecting the GGQ domain tend to be associated with more severe phenotypes, reinforcing a possible genotype-phenotype correlation.
\end{abstract}

Keywords: C12orf65, Leigh syndrome, COXPD7, mitochondrial, oxidative phosphorylation.

Received: October 15, 2018; Accepted: August 21, 2019.

Leigh syndrome is an early-onset progressive neurodegenerative disorder associated with defects of mitochondrial oxidative phosphorylation and is the most common distinct phenotype among oxidative phosphorylation disorders in children. The syndrome affects 1 in 32,000 - 40,000 newborn children (Rahman et al., 1996; Darin et al., 2001), but higher incidences up to 1 in 2000 have been reported due to founder mutations in the Faroe Islands and in an isolated population near Quebec, Canada (Carrozzo et al., 2007; Ruhoy et al., 2014). The clinical criteria for the diagnosis include progressive neurological disease with motor and intellectual developmental delay, signs, and symptoms of brain stem and/or basal ganglia disease, raised lactate levels in blood and/or cerebrospinal fluid, and characteristic neuroimaging and/or postmortem neuropathological changes (Baertling et al., 2014). The brain changes are almost identical in all patients, despite substantial clinical and genetic heterogeneity. The onset of symptoms is considered typical between 3 and 12 months of age (Thorburn et al., 2003). The prognosis of Leigh syndrome is generally

Send correspondence to Eduardo Perrone. Departamento de Genética Médica, Universidade Federal de So Paulo, Rua Botucatu 394, 04021-001 So Paulo, SP, Brazil. E-mail: duperrone@gmail.com. poor, with rapid deterioration of cognitive and motor functions resulting in death within months or a few years (Sofou et al., 2014). This syndrome is genetically highly heterogeneous as evidenced by the causal association with variants in more than 75 mitochondrial or nuclear genes (Lake et al., 2016).

The nuclear C12orf65 gene at chromosome 12q24.31 comprises three exons, with 501 base pairs in its protein-coding exons 2 and 3. It encodes a soluble mitochondrial matrix protein related to the family of class I peptide chain release factors (RFs), which interacts with the large ribosomal subunit to release the polypeptide chain from the P-site-bound peptidyl-tRNA (Huynen et al., 2012; Kogure et al., 2012).

The C12orf65 protein consists of 166 amino acids that include an RF-1 domain (residues 53-146) and a GGQ motif (residues 71-73). This protein does not coprecipitate with mitochondrial ribosomes (Richter et al., 2010) and has no peptidyl-tRNA hydrolase activity (Antonicka et al., 2010).

Pathogenic variants in the C12orf65 gene impair the mitochondrial oxidative phosphorylation system and are causative of combined oxidative phosphorylation deficien- 
cy 7 (COXPD7). To date, at least 11 pathogenic variants in this gene have been reported (Antonicka et al., 2010; Shimazaki et al., 2012; Buchert et al., 2013; Heidary et al., 2014; Spiegel et al., 2014; Tucci et al., 2014). Mutations were first described in two unrelated pedigrees affected by Leigh syndrome (LS; MIM \#256000) and Leigh-like syndrome (Antonicka et al., 2010).

The phenotypes associated with C12orf65 variants may be classified into three groups: mild, intermediate and severe, distinguished by disease severity, mortality, and the lack or presence intellectual disability (Spiegel et al., 2014). Here we describe a 2-year-old female patient with Leigh syndrome and a novel frameshift deletion pathogenic variant of the C12orf65 gene. We also review clinical and molecular aspects of the clinical phenotype caused by C12orf65 variants.

The 2-year-old girl was admitted to our hospital presenting with respiratory failure and sleepiness. Physical examination showed global hypotonia, developmental delay, and no dysmorphisms. She was the first child of healthy consanguineous parents, without pathological familiar history. Delivery was preterm via caesarean section because of foetal distress. Her weight was $1.720 \mathrm{~kg}$, height of $41.5 \mathrm{~cm}$, and an occipitofrontal circumference of $29 \mathrm{~cm}$. The Apgar scores immediately after delivery and at 5 minutes were 3 and 8 , so she was given neonatal resuscitation. She was observed in the neonatal care unit for the first three months of life because of respiratory distress episodes. Chromosome analysis showed a normal karyotype, abdominal and ophtamological exams were unremarkable. The patient was then referred to the paediatric unit after the respiratory distress episodes were treated. Her developmental milestones were incompletely informed: social smile and holding head at 3 months, sitting without support at 10 months, crawling at 11 months, and standing up with support and first words at 12 months of age. After the first year of life, the patient presented with failure to thrive and neurological regression was reported as she stopped grabbing toys and pointing objects and persons. At 2 years of age, she started episodes of diarrhoea and sleepiness evolving with respiratory failure at home and was taken to the emergency room for support and management. Her respiratory failure could not be resolved, so she was referred to the paediatric intensive care unit for better support. Her laboratory findings showed consecutive high levels of serum lactate $(21 \mathrm{mg} / \mathrm{dL}$ when admitted to our critical time intervention programme, 19 $\mathrm{mg} / \mathrm{dL}$ at day $7,54 \mathrm{mg} / \mathrm{dL}$ at day $14,69 \mathrm{mg} / \mathrm{dL}$ at day 19 , and $30 \mathrm{mg} / \mathrm{dL}$ at day 24 after admission; the reference lactic acid level is $4.5-14.4 \mathrm{mg} / \mathrm{dL}$ ). Metabolic investigations included tandem mass and urinary organic acid analyses, which were normal. Ophthalmological examination showed optic atrophy and echocardiography, abdominal and doppler sonography were normal. The electroencephalography suggested parieto-occipital epileptiform discharges, and brain magnetic resonance imaging (MRI) showed bilateral high intensity T2/Flair foci in the periventricular and peritrigonal white matter, internal capsule of the posterior limb and thalamus periphery; additionally, hyperintensities were observed in the cerebral peduncle, midbrain tegmentum, dorsal pons, and medulla oblongata, with a few areas of restricted diffusion, which is typical for mitochondrial disorders (Figure 1).

Empirical mitochondrial vitamin cocktail therapy, including thiamine, riboflavin, coenzyme Q10, biotin and L-carnitine, was initiated without clinically detectable improvement. Her treatment outcome was poor, with oxygen dependence, spastic tetraplegy, and severe developmental delay.

Clinical exome sequencing was then performed. Informed consent was obtained from the patient's parents, according to the ethical principles of the Declaration of Hel-
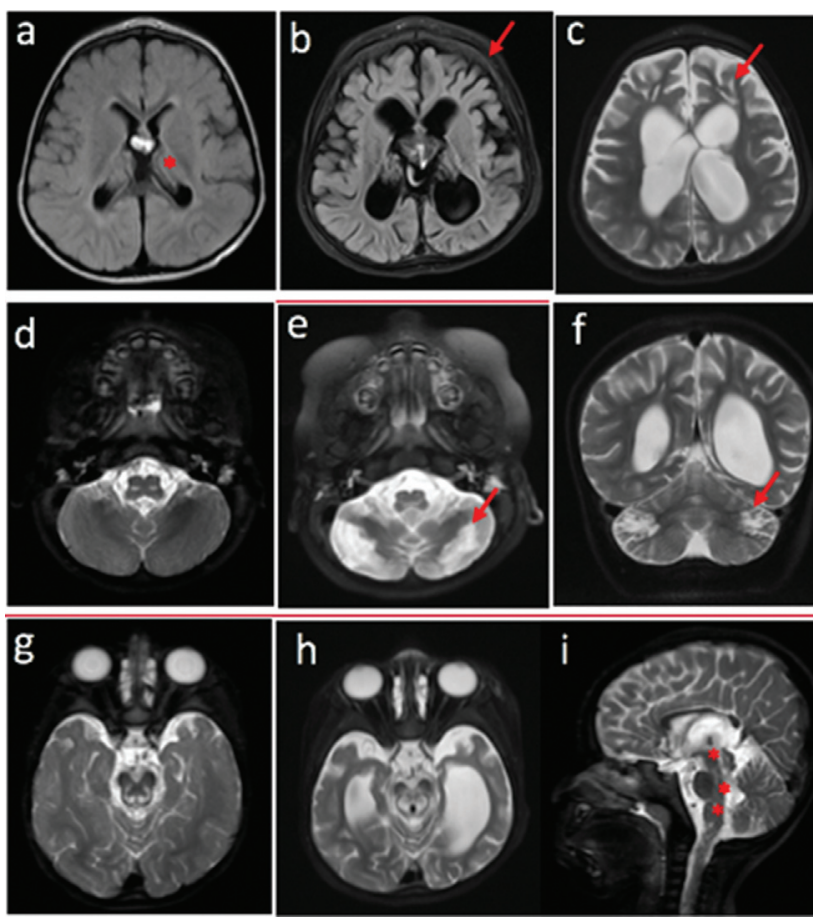

Figure 1 - Brain magnetic resonance imaging (MRI). Axial flair sequence MRI at 2 years (a) and 2 years and 9 months (b): A symmetrical and bilateral high-intensity signal is observed the periventricular and peritrigonal white matter in addition to the posterior arm of the internal capsule (red asterisk) and periphery of the thalamus; the accentuation of the sulci and encephalic cisterns results in diffuse encephalic reduction and compensatory ectasia of the supra and infratentorial ventricular system; the diploe is thickened (red arrow), a finding not observed before, which suggests progressive cerebral atrophy (b). Axial T2-weighted imaging at 2 years and 9 months (c) demonstrating symmetric focus with the same CSF sign in the two images in bilateral frontal white matter, suggesting enlargement of Virchow-Robin spaces, although lesion of cystic degeneration or encephalomalacia cannot be excluded. Axial T2-weighted imaging at ages 2 years (d) 2 years and 9 months (e), and coronal T2-weighted sequences at 2 years and 9 months of age (f): note the symmetrical high-intensity signal in the cerebellar hemispheres. Axial T2-weighted sequence at 2 years of age (g) and axial and sagittal T2-weighted imaging at age 2 years and 9 months $(h, i)$ : note the bilateral and symmetrical high-intensity signal of the substantia nigra of the cerebellar peduncle, mesencephalic segment, posterior region of the bridge, superior cerebellar peduncle and medulla oblongata. 
sinki. Genomic DNA was isolated from peripheral blood samples (QIAamp DNA Blood Mini Kit; Qiagen, Hilden, Germany), and was quantified by using the Qubit dsDNA HS Assay kit for the Qubit 2.0 Fluorometer (Life Technologies, Carlsbad, CA). We used the TruSight ${ }^{\mathrm{a}}$ One Sequencing Panel (Illumina, Inc., San Diego, CA, USA) for paired-end sequencing with a $\mathrm{v} 600$ cycles kit on a MiSeq System platform (Illumina), according to the manufacturer's guidelines. The FASTQ files were aligned to the Human Genome Reference Consortium build 37 (hg19) by using the Burroughs Wheeler Alignment algorithm as implemented in the BWA software package. After genome alignment, Variant Calling was performed by using the Genome Analysis Toolkit (GATK). Variants were annotated by using ANNOVAR, and selected read alignments were visualised by using the Integrative Genomics Viewer. All regions with a sequencing depth $<20$ were considered unsuitable for analysis. Furthermore, we established a minimum threshold Phred-like quality score of 30 (base call accuracy of $99.9 \%$ ). Common variants (3 1\% in the general population) were discarded by comparison with the 1000 Genomes, the Exome Variant Server, and the Exome Aggregation Consortium database. Synonymous variants were filtered and only exonic and splice site variants were prioritized. Initially, we evaluated the variants in the homozygous state, because the parents of the proband were consanguineous, and prioritized genes based on phenotypic databases, such as the Online Mendelian Inheritance in Man. The variant identified as pathogenic was confirmed by Sanger sequencing.

We identified a novel homozygous $14 \mathrm{bp}$ frameshift deletion in exon 2 of the C12orf65 gene: c.207_220del; p.Pro70Asnfs*28 (NM_152269), which was validated by Sanger sequencing, the sequence, being aligned with CRISP-ID (Figure S1). This variant is not present in population control databases (Exome Aggregation Consortium, 1000 genomes and Exome Variant Server). The truncated protein is predicted to have 96 aa as opposed to the original length of 166 aa, lacking the GCQ domain (residues 57-121) and the coiled-coil region (residues 127-160) (UniProt databank); this likely results in loss of function. Based on these features and according to the American College of Medical Genetics and Genomics guidelines, this variant is considered to be pathogenic (Richards et al., 2015).

To our knowledge, 27 patients were described with pathogenic variants in the C12orf65 gene with different clinical diagnoses (Table S1). The phenotype can vary from spastic paraparesis - type 55 - to Leigh syndrome. It has been thought that the main clinical features caused by C12orf65 variants correspond to a triad of optic atrophy, peripheral neuropathy, and spastic paraparesis, associated or not with Leigh syndrome. The information available from 27 patients showed that $92.3 \%$ presented with optic atrophy, $80 \%$ with peripheral neuropathy, $60 \%$ with spas- ticity, $52.1 \%$ with cognitive impairment, $42.8 \%$ with nystagmus, $38.4 \%$ with ophthalmoplegia, and $20 \%$ with bulbar disfunction. Our patient had optic atrophy, bulbar dysfunction (manifested as dysphagia and respiratory failure), and neurodevelopmental delay. We could not evaluate nystagmus and ophthalmoplegia, and spasticity was not present at the time of examination; the MRI findings were typical for Leigh syndrome. Our patient could be included in the COXPD7 severe phenotype, as she had a dramatically deteriorating course as described by Antonicka et al. (2010). This phenotype consists of significant cognitive and motor impairment and results in wheelchair dependence and typically abnormal Leigh syndrome brain MRI. Moreover, our patient showed a more severe phenotype when compared to patients described previously. The severity of this phenotype can be explained by the variant found, which affects an important functional protein domain (Figure 2).

Regarding the molecular status, the 27 described patients had 11 different pathogenic variants in the C12orf65 gene (Table S1). These variants were predominantly nonsense, splicing site and frameshift, and considered to be null variants. Only one of them, c.282G > A (p.Lys94Lys), was synonymous, but it was predicted to create a loss of splicing site with retention of intron 2 , thus acting as a frameshift variant. The variant found in our patient was also a null variant, and we suggest that truncated C12orf65 proteins were generated after the deletion of 14 base pairs, which reinforces the idea that a loss-of-function mechanism is a fundamental part of the pathogenesis.

We classified the phenotype of the reviewed patients as severe when there was bulbar dysfunction, or typical MRI findings of Leigh syndrome, or intellectual disability/neurodevelopmental delay, or absence of ambulation. The variants linked to severe phenotypes were more likely to affect the GCQ domain of the protein, although they could impact other protein domains (Figure 2).

The reviewing of clinical features and variants of 27 already described patients and the patient herein shows that the phenotype associated with C12orf65 variants is heterogeneous and that variants impacting the GGQ protein domain tend to be associated with more severe phenotypes. This is in agreement with Spiegel et al. (2014), who concluded that a clear genotype-phenotype correlation is anticipated, with deleterious mutations that disrupt the GGQcontaining domain being expected to result in a more severe phenotype, whereas downstream C-terminal alterations might result in a more favourable phenotype, typically lacking cognitive impairment.

Our review also showed that the median age for the onset of symptoms was 4 years and the median age for diagnosis was 17 years, so there was a 13-year diagnostic delay. Our patient presented the first symptoms immediately after birth, and the diagnosis was made two years later. A diagnostic delay may occur because of a variable phenotypic expression, despite the same aetiology (pathogenic variants 


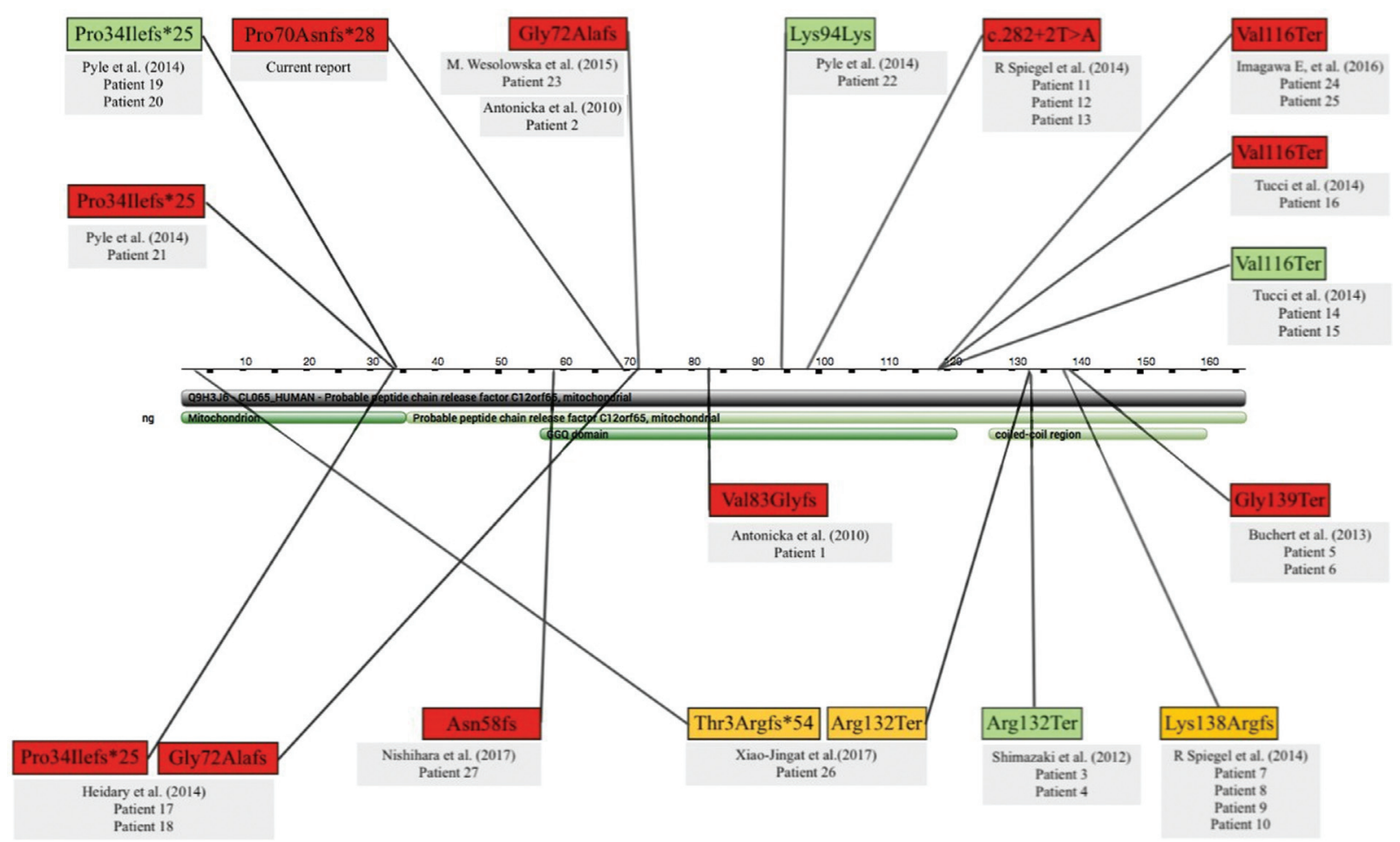

Figure 2 - Protein structure and distribution of variants in the C12orf65 gene. The yellow boxes represent the variants described in patients with mild phenotypes, the red boxes the variants in patients with severe phenotype, and the green boxes the variants in patients in whom it was not possible to classify disease severity based on the available clinical information. If just one variant is associated with one patient it was found in the homozygous state; two variants linked to one patient indicates compound heterozygosis. Patients are numbered as in Table S1.

in the C12orf65 gene), the rarity of the disease, the lack of knowledge about its existence and because of difficult access to molecular confirmation. Compared with literature data, our diagnostic delay was shorter, probably due to our patient having classical MRI signals of Leigh syndrome and elevated serum biomarkers (e.g., lactate), as well as a severe phenotype that contributed to active search for the aetiology. The prompt access to exome sequencing allowed a molecular diagnosis of the disorder.

In conclusion, the present study allowed the identification of a C12orf65 gene variant associated with a severe phenotype of Leigh syndrome caused by COXPD7 and reinforces the importance of the GGQ protein domain for the conserved peptidyl-hydrolase function. Furthermore, this study highlights that the mechanism of loss of function has an important role in the pathogenesis of the disease. Additional reports could help to better understand genotypephenotype correlations.

\section{Acknowledgments}

We thank GeneOne/DASA for providing financial support in performing the next-generation sequencing of the patient's sample.

\section{Conflict of Interest}

The authors have no conflicts of interest to disclose.

\section{Author Contributions}

EP conceived the study, wrote and reviewed the manuscript; TRC wrote and reviewed the manuscript; MGO, LAV, MFBS, SBOI, CSCM, MAC and CM reviewed the manuscript and contributed with clinical information; $\mathrm{AF}$, JSS, VN and MFM performed molecular analysis; MFFS contributed by describing the MRI. All authors read and approved the submitted version of the manuscript.

\section{References}

Antonicka H, Ostergaard E, Sasarman F, Weraarpachai W, Wibrand F, Pedersen AMB, Rodenburg RJ, van der Knaap MS, Smeitink JAM, Chrzanowska-Lightowlers ZM et al. (2010) Mutations in C12orf65 in patients with encephalomyopathy and a mitochondrial translation defect. Am J Hum Genet 87:115-22.

Baertling F, Rodenburg RJ, Schaper J, Smeitink JA, Koopman WJH, Mayatepek E, Morava E and Distelmaier F (2014) A guide to diagnosis and treatment of Leigh syndrome. J Neurol Neurosurg Psychiatry 85:257-65.

Buchert R, Uebe S, Radwan F, Tawamie H, Issa S, Shimazaki H, Henneke M, Ekici A, Reis A and Jamra RA (2013) Mutations in the mitochondrial gene C12ORF65 lead to syndromic autosomal recessive intellectual disability and show genotype phenotype correlation. Eur J Med Genet 56:599-602.

Carrozzo R, Dionisi-Vici C, Steuerwald U, Lucioli S, Deodato F, Giandomeninco S, Bertini E, Franke B, Kluijtmas LA, Meschini MC et al. (2007) SUCLA2 mutations are associated 
with mild methylmalonic aciduria, Leigh-like encephalomyopathy, dystonia and deafness. Brain 130:862-74.

Darin N, Oldfors A, Moslemi AR, Holme E and Tulinius M (2001) The incidence of mitochondrial encephalomyopathies in childhood: Clinical features and morphological, biochemical, and DNA abnormalities. Ann Neurol 49:377-83.

Heidary G, Calderwood L, Cox GF, Robson CD, Teot LA, Mullon J and Anselm I (2014) Optic atrophy and a Leigh-like syndrome due to mutations in the c12orf65 gene: Report of a novel mutation and review of the literature. J Neuroophthalmol 34:39-43.

Huynen MA, Duarte I, Chrzanowska-Lightowlers ZM and Nabuurs ZM (2012) Structure based hypothesis of a mitochondrial ribosome rescue mechanism. Biol Direct 7:14.

Kogure H, Hikawa Y, Hagihara M, Tochio N, Koshiba S, Inoue Y, Guntert P, Kigawa T, Yokoyama S and Nameki N (2012) Solution structure and siRNA-mediated knockdown analysis of the mitochondrial disease-related protein C12orf65. Proteins 80:2629-42.

Lake NJ, Compton AG, Rahman S and Thorburn DR (2016) Leigh syndrome: One disorder, more than 75 monogenic causes. Ann Neurol 79:190-203.

Rahman S, Blok RB, Dahl HH, Danks DM, Kirby DM, Chow CW, Christodoulou J and Thorburn DR (1996) Leigh syndrome: clinical features and biochemical and DNA abnormalities. Ann Neurol 39:343-51.

Richards S, Aziz N, Bale S, Bick D, Das S, Gastier-Foster J, Grody WW, Hegde M, Lyon E, Spector E et al. (2015) Standards and guidelines for the interpretation of sequence variants: a joint consensus recommendation of the American College of Medical Genetics and Genomics and the Association for Molecular Pathology. Genet Med 17:405-24.

Richter R, Rorbach J, Pajak A, Smith TM, Wessels HJ, Huynen MA, Smeitink JA, Lightowlers RN and ChrzanowskaLightowlers S (2010) A functional peptidyl-tRNA hydrolase, ICT1, has been recruited into the human mitochondrial ribosome. EMBO J 29:1116-25.

Ruhoy IS and Saneto RP (2014) The genetics of Leigh syndrome and its implications for clinical practice and risk management. Appl Clin Genet 7:221-34.

Shimazaki H, Takiyama Y, Ishiura H, Sakai C, Matsushima Y, Hatakeyama H, Honda J, Sakoe K, Naoi T, Namekawa M et al. (2012) A homozygous mutation of C12orf65 causes spastic paraplegia with optic atrophy and neuropathy (SPG55). J Med Genet 49:777-84.
Sofou K, De Coo IF, Isohanni P, Ostegaard E, Naess K, Meirleir L, Tzoulis C, Uusimaa J, Angst IB, Lönnkvist T et al. (2014) A multicenter study on Leigh syndrome: disease course and predictors of survival. Orphanet J Rare Dis 9:52.

Spiegel R, Mandel H, Saada A, Lerer I, Burger A, Shaag A, Shalev SA, Jabali-Habib H, Goldsher D, Gomori JM et al. (2014) Delineation of C12orf65-related phenotypes: a genotype-phenotype relationship. Eur J Hum Genet 22:1019-25.

Tucci A, Liu YT, Preza E, Priceathly RDS, Chalasani A, Plagnol V, Land JM, Trabzuni D, Ryten M, UKBEC et al. (2014) Novel C12orf65 mutations in patients with axonal neuropathy and optic atrophy. J Neurol Neurosurg Psychiatry $85: 486-92$.

\section{Internet Resources}

CRISP-ID, http://crispid.gbiomed.kuleuven.be (accessed 1 August 2018).

Exome Aggregation Consortium database, http://exac.broadinstitute.org (accessed 1 August 2018).

Exome Variant Server, http://evs.gs.washington.edu (accessed 1 August 2018).

Online Mendelian Inheritance in Man, http://omim.org/ (accessed 1 August 2018).

UniProt data bank, http:// http://www.uniprot.org (accessed $1 \mathrm{Au}-$ gust 2018).

Thorburn DR, Rahman J, Rahman S (2003) Mitochondrial DNA-Associated Leigh Syndrome and NARP, https://www.ncbi.nlm.nih.gov/books/NBK1116/ (accessed 1 August 2018).

24.1000 Genomes, http://www.1000genomes.org (accessed 1 August 2018).

\section{Supplementary material}

The following online material is available for this article: Table S1 - Clinical and molecular features of 27 patients with $C 12$ orf65 pathogenic variants from the literature and the present case.

Figure S1 - Alignment of sequence in CRISP-ID V1.1a.

Associate Editor: Angela M. Vianna-Morgante

License information: This is an open-access article distributed under the terms of the Creative Commons Attribution License (type CC-BY), which permits unrestricted use, distribution and reproduction in any medium, provided the original article is properly cited. 